\title{
Nathaniel Hawthorne et les voix du passé
}

\section{Kathie Birat}

\section{(2) OpenEdition}

Journals

Édition électronique

URL : https://journals.openedition.org/clo/1861

DOI : $10.4000 /$ clo. 1861

ISSN : 2266-1816

Éditeur

INALCO

\section{Édition imprimée}

Date de publication : 1 janvier 2014

ISBN : 978-2-85831-222-1

ISSN : 0396-891X

\section{Référence électronique}

Kathie Birat, " Nathaniel Hawthorne et les voix du passé », Cahiers de littérature orale [En ligne], 75-76 |

2014, mis en ligne le 27 avril 2015, consulté le 30 juin 2021. URL : http://journals.openedition.org/clo/ 1861 ; DOI : https://doi.org/10.4000/clo.1861

Ce document a été généré automatiquement le 30 juin 2021.

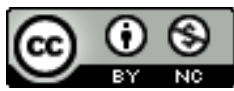

Cahiers de littérature orale est mis à disposition selon les termes de la Licence Creative Commons Attribution - Pas d'Utilisation Commerciale 4.0 International. 


\title{
Nathaniel Hawthorne et les voix du passé
}

\author{
Kathie Birat
}

Avec la publication de la Lettre écarlate en 1850, Nathaniel Hawthorne écrit l'un des textes fondateurs de la littérature américaine. Mais cette œuvre romanesque, qui prend comme sujet une lettre brodée sur la robe d'une femme adultère, est également un texte-clé pour comprendre la relation entre l'écrit et l'oral dans l'histoire culturelle des États-Unis. Comment la critique pourrait-elle résister à la tentation de «broder » sur cette histoire dense et passionnelle, puisqu'elle tourne autour de la question complexe du rapport entre la lettre censée fixer la vérité une fois pour toutes aux yeux de tous et la prolifération des interprétations qu'elle va déclencher chez les citoyens de Salem? Alessandro Portelli voit cette relation instable entre l'écrit et l'oral comme l'un des thèmes principaux de la littérature américaine depuis ses débuts. Pour Portelli, dont l'ouvrage The Text and the Voice porte le sous-titre Writing, Speaking and Democracy in American Literature, parler d'oralité dans le contexte américain implique la nécessité de «rompre le lien romantique entre la voix, le peuple, et l'identité nationale (Portelli, 1994, 30, ma traduction). L'oralité, dit Portelli, « donne la voix à la démocratie, mais mine subrepticement les institutions nationales en nourrissant les souvenirs, les rites, les rassemblements et les passions qui échappent au contrôle et aux certitudes de la raison écrite et la loi " (Portelli, 1994, 31, ma traduction) ${ }^{1}$. L'analyse de Portelli est ambitieuse, embrassant, dans une perspective nourrie par la lecture des textes majeurs sur l'oralité, la totalité de la littérature américaine. Elle reflète une approche de l'histoire culturelle des États-Unis qui a beaucoup influencé la lecture des grands classiques à partir des années $1980^{2}$. L'analyse proposée ici se donne un objectif plus modeste, qui consiste à tisser quelques liens, aussi fragiles soient-ils, entre l'expérience qu'aurait pu faire Hawthorne d'une culture orale au début du XIXe siècle et l'écriture qu'il produisit à partir de ses premiers contes, dont la publication dans des périodiques de l'époque date des années $1830^{3}$. L'intérêt de porter un regard sur Hawthorne par le biais de l'oralité repose sur la nature quelque peu paradoxale d'une telle entreprise. En dépit de la place centrale que Portelli accorde à Hawthorne dans sa démonstration, le rôle joué par l'oralité dans l'œuvre d'Hawthorne ne va pas de soi. À la différence 
d'Herman Melville et de Mark Twain, Hawthorne ne voyait pas dans l'échange oral, dans la construction collective des récits, la source de la vérité qu'il cherchait à capter ${ }^{4}$. À l'image de l'un de ses personnages les plus célèbres, l'artiste du conte «l'Artiste du beau » qui s'épuise à essayer de produire une image parfaite de la beauté en fabriquant un papillon mécanique, Hawthorne représentait souvent le commerce humain, le dialogue, comme la source d'une contamination incompatible avec le beau et le vrai. Mais l'artiste du conte ne montre qu'une facette d'une œuvre qui fut construite en partie par la prise en compte d'une réalité hétérogène, désordonnée, qui s'exprimait souvent à travers le langage de tous les jours, dans les anecdotes racontées par des personnes rencontrées dans des tavernes et les auberges, ou dans les livres d'histoire dont Hawthorne notait dans ses carnets les passages qui attiraient son attention. Regarder Hawthorne dans la perspective de l'oralité, c'est effectuer un rapprochement entre les traces d'une expérience de l'oralité et les thématiques apparemment si éloignées du monde de l'oral que l'on trouve dans beaucoup de ses textes. Le but d'une telle démarche est de montrer comment son expérience d'une culture orale aurait pu suggérer à l'auteur une manière d'aborder certaines thématiques en révélant leur lien avec l'oralité, des liens qui ne sont pas toujours évidents d'un premier abord.

2 Le terme "oralité », tel qu'il est utilisé dans cette étude, fait référence à la fois aux récits qui relèvent d'une tradition orale, comme les contes, les mythes et les légendes, et à la production d'un récit dans un contexte d'oralité, qu'il s'agisse d'un récit nouveau, inventé par l'énonciateur, ou de la reprise d'un récit existant. Nous considérons qu'Hawthorne s'intéressait à la fois au contenu des contes et des légendes, et aux conditions de création et de transmission de ces récits. Pour bien comprendre son rapport à l'oralité, il faut tenir compte de la nature hybride de la littérature orale que connaissait Hawthorne, car il fut dès l'âge de quatre ans un lecteur vorace, et il découvrit une grande partie de la littérature d'origine orale à travers ses lectures. Mais il rencontra également l'oralité directement dans le contexte familial.

\section{Écoute et lecture : l'arrière-plan culturel}

3 Hawthorne grandit dans une famille dont l'histoire remontait à l'époque du Massachusetts Bay Colony de John Winthrop. Le fils de ce premier habitant de Salem, John Hathorne ${ }^{5}$, fut l'un des juges lors du procès des sorcières de Salem ; ce dernier fut l'arrière-grand-père du romancier. Nathaniel Hawthorne, comme le souligne l'un de ses biographes, «fut fasciné par ces régions obscures de l'esprit humain qui avaient été révélées au sein du tribunal que présidait son ancêtre du dix-septième siècle » (Mellow, 1980, 11, ma traduction). Dans une lettre envoyée à Samuel Goodrich en 1829, l'auteur évoqua, à propos d'une série de contes qu'il espérait faire publier, la façon dont son imagination avait été frappée par les superstitions concernant les sorcières et autres phénomènes surnaturels qui figuraient dans les légendes orales de la NouvelleAngleterre :

Vous verrez que l'une des histoires est basée sur les superstitions que l'on trouve dans cette région. Je ne sais pas si une telle chose a été tentée auparavant, mais comme j'ai perdu beaucoup de temps à écouter de telles traditions, je n'ai pas pu résister à la tentation de leur donner une forme. Le conte est assez extravagant et grotesque certes, mais on peut en entendre pas mal qui ne le sont pas moins dans ce coin du pays. (Woodson, Smith and Pearson, 1984, 199, ma traduction). 
Cette lettre établit clairement un rapport entre ses sources d'inspiration et le fonds légendaire dans lequel sa propre famille plongea ses racines. Ce lien entre les dimensions privées et publiques de son rapport au passé américain explique en partie la façon particulière dont l'auteur représentait ce passé dans sa fiction. Jane Lundblad fait remarquer qu'Hawthorne découvrit le passé de sa famille en partie à travers les histoires que sa tante maternelle lui raconta (Lundblad, 1965, 33). Brenda Wineapple, dans une biographie récente, explique qu'en plus d'un penchant pour la lecture des livres d'histoire, il s'intéressa aux traces concrètes du passé, comme les livres de cuisine, ou les épingles exposées au tribunal de Salem, dont on disait qu'elles avaient servi aux sorcières, «qui les enfonçaient dans la chair de leurs victimes " (Wineapple, 2003,61 , ma traduction). Son père joua également un rôle dans le développement d'une sensibilité à la narrativité orale. Hathorne père fut capitaine d'un bateau (ou plutôt de plusieurs bateaux) jusqu'à sa mort au cours d'un voyage dans les Antilles en 1808 quand son fils avait quatre ans. Luedtke cite l'auteur d'un article consacré au père d'Hawthorne qui affirme que le jeune Hawthorne, loin d'être isolé du monde réel, grandit au milieu des activités maritimes de Salem, «littéralement au bord de l'eau, parmi les bateaux et les marins et les histoires de la mer » (Hoeltje, 1953, 332, cité dans Luedtke, 1989, 22, ma traduction). Plus tard, pendant son séjour au bureau de la douane à Salem comme inspecteur, il nota dans son carnet l'histoire d'une immense tortue aperçue par le capitaine Burchmore lors d'un voyage à Batavia. Il ajouta en conclusion :

Le vieux Lee vint confirmer cette histoire en certifiant qu'il avait souvent entendu d'autres commandants de navire parler de ce même monstre, mais comme c'est un menteur notoire et que le capitaine Burchmore aime raconter, sans le moindre scrupule, des histoires interminables et des aventures invraisemblables, le témoignage ne vaut que pour ce qu'il est. (Hawthorne, 1995a, 364-65)'.

Comme nous l'avons déjà signalé, dès son plus jeune âge Hawthorne lisait abondamment, et même sa connaissance des voyages de son père fut acquise par la lecture de ses journaux de bord (Luedtke, 1989, 5-15). En envisageant le rôle de l'oralité dans l'éducation littéraire du jeune Hawthorne, il est donc indispensable de tenir compte de ses lectures nombreuses et variées?. Il avait lu le Pilgrim's Progress de Bunyan très jeune (dès l'âge de quatre ans d'après Lundblad, 1965, 33) ainsi que la Fairie Queene de Spenser et les pièces de Shakespeare. Mais ses lectures furent également conformes à ce qu'on pouvait attendre d'un garçon à l'époque; il dévora les romans de Walter Scott et les romans gothiques de Mme Radcliffe et d'Horace Walpole, il avait lu les Mille et Une Nuits avant l'âge de quinze ans ${ }^{8}$. Son goût de l'exotisme, qui explique en partie la lecture des journaux de son père, le poussa également à lire de nombreux récits de voyage, un genre très en vogue à l'époque'. Il est également important de tenir compte des livres d'histoire, en particulier ceux concernant l'histoire de la NouvelleAngleterre, comme les Annals of Salem de Felt et les papiers de Thomas Hutchinson, le gouverneur du Massachusetts Bay Colony qui avait fui pour se réfugier en Angleterre juste avant la Révolution. Il lut en détail les documents concernant la justice pénale en Angleterre rassemblés par Howells. Et enfin il lut le Magnalia Christi de Cotton Mather, l'histoire monumentale de la Nouvelle-Angleterre puritaine rédigée par l'un des pasteurs les plus connus. 


\section{L'écriture et la vie : les Carnets américains}

6 En lisant les contes d'Hawthorne, en particulier les plus connus comme « Maître Brown fils", "le Voile noir du pasteur" ou "la Marque de naissance», on peut avoir l'impression qu'Hawthorne trouvait ses modèles dans les contes de fées ou dans les mythes; le sens semble dépendre en large partie de la forme, et l'univers du conte a l'air de se trouver loin de la réalité quotidienne de l'époque. Les titres mêmes, avec l'emploi de l'article défini, font l'effet de fables, d'allégories, de paraboles: «l'Homme de diamant, ", «le Doux Enfant, " «le Glas nuptial, " "l'Ambitieux Convive» sont autant d'exemples frappants. La vie américaine de l'époque semble n'être présente que de façon oblique et figurale. Le rapport complexe que l'auteur entretenait avec le monde qui l'entourait, rapport soigneusement décrit et élaboré dans les préfaces de ses romans, ne doit pas pour autant cacher la persistance d'un lien fort avec son environnement immédiat ${ }^{10}$. La lecture de ses carnets et de sa correspondance révèle le nombre et la fréquence de ses contacts et son intérêt pour les détails de la vie américaine (Stewart, 1932, XLII). Une lettre envoyée en 1837 au poète Henry Wadsworth Longfellow, qu'il avait connu pendant ses études universitaires à Bowdoin College dans le Maine, montre le souci d'Hawthorne d'ancrer sa fiction dans la vie réelle: "Le manque de matériaux», dit l'auteur, "constitue une autre difficulté majeure pour moi ; j'ai vu si peu du monde, que mes histoires semblent être concoctées à partir de l'air pur, et il n'est pas facile de donner un semblant de vie à une matière si peu substantielle " (cité dans Woodson, Smith et Pearson, 1984, 252, ma traduction). Dans les Carnets, les descriptions de la nature ou des personnes rencontrées lors de voyages alternent avec des idées pour des contes. On trouve, par exemple, pour le 16 juillet 1837, le commentaire suivant :

Description de différentes catégories d'hôtels et de tavernes, ainsi que des personnages importants qui s'y trouvent. Il faudrait une histoire pour relier le tout, comme, par exemple, quelqu'un qui descendrait dans un grand hôtel et qui, au fur et à mesure que ses moyens diminueraient, se trouverait de plus en plus contraint, jusqu'à se retrouver dans une cave en sous-sol. (Hawthorne, 1995a, 98).

7 La vie ordinaire et son potentiel comme source d'une littérature ancrée dans la vie américaine est très présents dans les Carnets. Sa description d'une exposition de figures en cire dans une fête foraine fournit une illustration intéressante de son observation de la vie américaine à travers les divertissements populaires, toujours très révélateurs des caractéristiques d'une société en mutation comme celle du début du XIX siècle. Il décrit les personnages représentés, "dans la grande majorité des meurtriers et leurs victimes » (Hawthorne, 1995a, 115). Il présente également le forain :

C'est un être qui a les manières aisées et affables d'un homme presque distingué, dont la façon de parler a subi l'influence des personnes qui fréquentent habituellement ce genre de spectacle, qui affecte l'air supérieur de celui qui sait, du donneur de leçons de morale, le tout mêlé d'une bonne dose de réelle expérience du monde. (Hawthorne, 1995a, 116).

8 Il fait le portrait de l'un des spectateurs : "Il y a là un individu mi-rustre, migentilhomme campagnard, qui a l'air de quelqu'un qui sait, mais qui, pourtant, regarde et écoute avec beaucoup de simplicité et de bonne foi en souriant de temps à autre » (Hawthorne, 1995a, 117). Il termine par se représenter lui-même comme participant de la scène : «il y a enfin un garçon ou deux et puis moi-même, qui examinons les figures de cire et les figures de chair avec un égal intérêt» (Hawthorne, 1995a, 117). Dans ce 
passage, on voit surtout l'intérêt d'Hawthorne pour le lien entre une société et ses représentations, le comportement des gens, leur façon de s'habiller, de se tenir, et de parler faisant partie de ces représentations autant que les sujets des statues de cire.

Les Carnets ont été utilisés de différentes façons par les chercheurs dans l'étude de la fiction d'Hawthorne. Alors que l'une des premières études des sources de ces contes, celle d'Elizabeth Chandler, tente d'établir une relation entre les contes et la correspondance et carnets de l'auteur, Milder et Fuller considèrent «le style et le contenu picaresques des journaux de voyage " comme " une direction qu'Hawthorne n'a pas suivie, même s'ils représentent l'homme Hawthorne et l'écrivain autant que "l'Arbre de mai de Merrymount" et "le Voile noir du pasteur" " (Milder et Fuller, 2009, 9, ma traduction). En regardant plus attentivement, on s'aperçoit que la ligne de partage entre les détails puisés dans la vie américaine et la nature allégorique des contes ne suit pas forcément la distinction entre «les descriptions de la réalité quotidienne » et « les notations brèves pour des contes et des esquisses » mentionnée par Milder et Fuller (Idem, 2, ma traduction). Le rapport que l'auteur voyait entre la surface de la réalité américaine et le monde imaginaire qu'il tentait de créer était plus complexe.

\section{Passé et oralité : les premiers contes}

La lecture des premiers contes d'Hawthorne permet d'observer les tentatives de l'auteur pour utiliser le fonds légendaire auquel il fait allusion dans sa correspondance avec Goodrich. Avant même d'avoir terminé ses études à Bowdoin, Hawthorne avait écrit une série de contes intitulée Sept contes de mon pays natal. Ces contes ne furent jamais publiés, car l'auteur les brûla dans un moment de doute avant même qu'ils ne fussent publiés. Selon sa sœur Elizabeth, ce recueil tournait autour « des sorcières, des pirates et des corsaires " (Luedtke, 1989, 104, ma traduction) ${ }^{11}$. En 1829, il envoya à Goodrich un deuxième recueil qui contenait des histoires situées à l'époque des premières colonies et de la Révolution américaine et qu'il projeta d'appeler Contes provinciaux. Il aurait voulu les publier comme un ensemble, mais Goodrich, éditeur d'une revue annuelle, The Token, finit par le convaincre de le laisser en publier certains dans sa revue ${ }^{12}$. Découragé par l'échec de son projet de publier les contes sous forme de recueil, il entama l'écriture d'un troisième ensemble qui portait le titre de The Story Teller $^{13}$. Les histoires et esquisses devaient être encadrées et liées par le discours d'un conteur itinérant. Comme le fait remarquer Luedtke, la structure envisagée par Hawthorne fut inspirée des modèles de récits enchâssés empruntés à la littérature orientale et permettait de relier l'Amérique contemporaine du conteur au passé raconté dans certaines des histoires (Luedtke, 1989, 105). À travers ce projet, Hawthorne semble se rapprocher d'un modèle exploité par d'autres auteurs américains, comme Washington Irving et Henry Wadsworth Longfellow, en utilisant une oralité inventée pour profiter de l'engouement du public pour la littérature orientale et répondre au désir des lecteurs de découvrir un patrimoine légendaire américain. Mais la lecture du morceau «les Sept Vagabonds », prévu comme introduction au recueil, révèle un rapport particulier au modèle. Le narrateur présente sa rencontre avec plusieurs personnages hauts en couleur, dont un «montreur d'automates", un couple qui se promène avec une "boîte de curiosités ", un mendiant, un Peau-Rouge et "un homme de lettres ", «l'auteur véritable d'un livre imprimé » (Hawthorne, «les Sept 
Vagabonds » dans Monsieur du miroir, 1992, 27). Leur destination est un " campement de la mission " (Hawthorne, Idem, 27), dans la ville de Stamford. Hawthorne trace, par la voix du narrateur, une série de portraits stéréotypés, déclarant qu'il ne partage pas les préjugés d'un «Yankee pur sang " (Hawthorne, Idem, 37) et imaginant que le PeauRouge préfère la solitude à la compagnie des autres. Mais son ironie ne vise pas seulement les distractions populaires ou les travers de son époque. Par le biais du narrateur qui rêve de devenir " un romancier itinérant » (Hawthorne, Idem, 49) il crée un oxymore amusant qui interroge le rapport entre l'écrit et l'oral, entre l'artiste solitaire et la culture populaire. Se trouve posée ainsi, quoique de façon oblique, la question qui le taraude depuis le début de sa carrière, celle de sa place dans la société et de son rapport à la vie réelle de son époque. Se trouvent parmi les marchandises du «montreur d'automates» vantées par ses talents de vendeur, les textes écrits qui avaient nourri son imagination d'enfant, «les romans des jours heureux où mon affection balançait entre les chefs de clans écossais et Tom Pouce » (Hawthorne, Idem, 25). Revalorisés par le discours du vendeur et rendus attractifs par l'ambiance festive du spectacle ambulant, ces textes redeviennent des objets de désir et de convoitise :

Je fus heureux de trouver ce cher et vénérable petit volume, le Livre de lecture de la Nouvelle-Angleterre, qui avait l'air plus antique que jamais, bien qu'il fût dans sa millième nouvelle édition. Une pile de livres dorés sur tranche et surannés fit de moi un tel enfant que, en partie pour leurs couvertures étincelantes, et en partie pour les contes de fées qu'ils contenaient, j'achetai le tout. (Hawthorne, « Les sept vagabonds », 1992, 25-26).

Les contes de fées faisant appel à une tradition orale, l'écrit et l'oral se trouvent ainsi pris dans une spirale qui révèle leur imbrication profonde. Mais le rôle de l'écrivain est également mis dans la balance, car face à "l'auteur véritable", qui voyage avec le spectacle, emportant avec lui un certain nombre de livres, le narrateur découvre une autre façon de faire "circuler» les textes écrits, un moyen plus participatif, plus convivial que la création solitaire des «rats de bibliothèques" comme lui-même. Malgré le ton léger et humoristique, ce texte pose la question de la place de l'oralité dans la création et la diffusion des textes à une époque où la lecture était en train de devenir une distraction privée et individuelle et où en même temps la nation réclamait une culture spécifique et nationale, ancrée dans l'histoire et le folklore des États-Unis.

La façon dont l'auteur utilise l'artifice de la narration « orale » dans ses premiers contes révèle une approche nuancée du folklore américain et du rôle de la littérature dans sa mise en valeur. La valorisation d'un patrimoine folklorique était l'un des enjeux à une époque où la littérature américaine était en train de définir sa spécificité, en particulier par rapport à la littérature anglaise. Washington Irving considérait le folklore et la légende comme les moyens « de lier le cœur du nouveau venu à sa patrie » et face à la pénurie de récits natifs, il adapta des contes allemands au contexte américain ${ }^{14}$. Hawthorne, pour sa part, ne se contenta pas de solliciter le folklore pour forger un passé mémoriel capable de générer l'adhésion à une mythologie de la patrie et à une identité nationale. Au contraire, s'il puisait dans ce fonds légendaire, c'était en partie pour interroger les conditions dans lesquelles ces récits furent inventés et pour les recréer d'une façon qui mettrait en évidence la complexité du rapport entre passé et présent.

En regardant l'un des premiers contes écrits par Hawthorne, "l'Invocation d'Alice Doane ", qui est certainement une version remaniée de l'un de ceux qui composaient le premier recueil disparu, on peut constater l'impact du cadre narratif sur la nature même du récit censé être puisé dans le passé puritain de Salem. L'histoire 
en elle-même est étrange et lugubre ${ }^{15}$ : le frère d'Alice, Léonard, aurait tué Walter Brome, qu'il soupçonne d'être son frère jumeau, parce qu'il pensait que ce dernier avait séduit Alice. Ce meurtre, dont le narrateur laisse supposer qu'il a été suggéré à Léonard par un sorcier qu'il avait consulté à propos de ses soupçons, donne au narrateur l'occasion de décrire un rassemblement de fantômes, constitué « des âmes damnées et des démons se donnant une apparence de sainteté » (Hawthorne, 1989, 276) qui sont venus au cimetière où Alice se trouve avec Léonard " pour fêter la découverte d'un crime obscur, plus vil qu'aucun crime jamais conçu en cet horrible séjour " (Hawthorne, Idem, 1989, 277) (c'est-à-dire, le crime commis par Léonard). Le véritable enjeu de ce conte est le pouvoir du narrateur à raconter le passé. Il comporte plusieurs récits enchâssés : l'histoire d'Alice Doane est racontée par le narrateur principal, qui le lit à deux amies qu'il amène en promenade sur la Colline des potences à Salem. Dans le cadre de l'histoire d'Alice, son frère devient à son tour narrateur quand il raconte au sorcier les raisons pour lesquelles il a tué Walter Brome. Dans la mesure où le narrateur laisse entendre que le meurtre est le travail du sorcier, il en fait également un narrateur capable d'avoir entraîné Léonard dans le péché. Ce qui importe est la manière dont le passé est mis en récit par chaque narrateur, car c'est de cela que dépend l'impact sur l'auditeur. Le but du narrateur premier est de faire comprendre à ses jeunes amies toute l'horreur de la chasse aux sorcières, «l'acte le plus abominable dont notre histoire ait à rougir " (Hawthorne, 1989, 264). Mais il affirme aussi que les Américains sont un peuple « qui vit dans le présent et qui n'éprouve nul intérêt sincère pour les temps passés » (Hawthorne, Idem, 265), ce qui explique sa frustration quand ses jeunes amies rient en entendant une histoire «trop grotesque et extravagante " pour leur faire peur (Hawthorne, Idem, 278). Vexé par cette réaction, il reprend la description de la foule de fantômes sur la colline, les désignant cette fois-ci comme les victimes de la chasse aux sorcières, et de leurs accusateurs, parmi lesquels figure Cotton Mather « fier de son titre bien mérité de représentant de tout ce qui dans son époque était haïssable » (Hawthorne, 1989, 280). En dépit de la dimension grotesque de ce conte de jeunesse, qui est loin d'être l'un des plus réussis, on perçoit clairement l'importance pour le narrateur de cette évocation des légendes. Son récit est en partie déterminé par la recherche d'une réaction, par le désir de toucher le cœur même de ses auditrices, comme si la compréhension du passé en dépendait (Baker, 2007, 109-111). Cette dimension est clairement mise en évidence par Lauren Berlant, qui analyse ce conte dans la perspective de l'élaboration d'une symbolique nationale à l'époque d'Hawthorne. Elle explique en particulier que ce conte montre à quel point l'auteur savait "que pour qu'un conte devienne le véhicule d'une véritable conscience historique, le texte doit d'une façon ou d'une autre créer la scène contemporaine qui "reçoit" les éléments culturels transmis » (Berlant, 1991, 37, ma traduction). Se trouve enfouie dans l'enchâssement même des récits la relation ambiguë à la narration orale, car les événements qui sous-tendent le récit, ceux liés à la persécution des sorcières, étaient le fruit également de légendes, de rumeurs, et d'histoires transmises de bouche à oreille. Hawthorne ne fit qu'amplifier des voix qui résonnaient déjà dans la colonie puritaine ${ }^{16}$. 


\section{Oralité et mémoire collective : « la Sépulture de Roger Malvin »}

14 La relation d'Hawthorne à l'oralité, aux légendes historiques et familiales qu'il entendit dans son enfance, ne fut pas un rapport figé, artificiel, nourri par un romantisme désireux de se fixer sur des objets trouvés dans la réalité américaine. Il utilisa parfois sa connaissance des légendes du pays pour enrichir l'évocation d'un lieu selon une méthode typique de son époque (Bell, 1971, 195-196) ${ }^{17}$. Mais sa fascination pour le passé fut sombre et passionnelle et finit par influencer les chefs-d'œuvre qu'il créa à partir de la fin des années 1840 et notammentla Lettre écarlate. C'est dans les contes, surtout ceux du début, qu'on peut le mieux observer le développement d'un traitement littéraire de ce fonds légendaire.

«La Sépulture de Roger Malvin» fournit une illustration intéressante de l'inscription de la culture produite et transmise par l'oralité dans une situation narrative qui semble à première vue posséder la simplicité d'une parabole biblique. Ce conte montre toute l'économie de moyens qui caractérise les récits les mieux réussis. Après la bataille de Lovewell, au cours de laquelle les Indiens Pequawket ont été battus, deux soldats tentent de regagner leur village. Le plus âgé, mortellement blessé, exhorte son compagnon, Reuben Bourne, à partir sans lui. Pour le convaincre, il lui explique qu'il pourra lui ramener de l'aide. Reuben finit par partir et retrouve le village et son amie, Dorcas, la fille du compagnon abandonné. Honteux d'avoir laissé Roger Malvin seul sans sépulture, Reuben n'ose pas parler de ce qui s'est vraiment passé et laisse entendre qu'il a fermé les yeux de Roger Malvin, ce qui est loin d'être le cas. Reuben épouse Dorcas, lui donne un fils et continue à vivre, rongé par la culpabilité. Des années plus tard, sans comprendre pourquoi, Reuben quitte la communauté et amène sa famille avec lui dans la forêt sauvage à la recherche d'une vie nouvelle. Sans qu'il s'en rende compte, c'est vers le lieu où il avait abandonné son compagnon que ses pas l'amènent. Entendant un bruit soudain, Reuben tire; en s'approchant il découvre que c'est son fils qu'il a tué exactement à l'endroit où se trouve le corps de Roger Malvin. Ce conte semble être une histoire de lâcheté et de punition; la coïncidence qui provoque la punition pourrait sortir directement d'une vision puritaine du monde, dans laquelle les événements correspondent au dessein d'un Dieu qui punit sévèrement ceux qui ne respectent pas la loi de la communauté. Le fait de ne pas avoir enterré Roger Malvin constitue non seulement un mensonge, mais un manquement grave aux rites les plus essentiels qui consacrent le passage entre la vie et la mort au sein de la communauté. On peut remplacer le déterminisme religieux par un autre, psychologique, et faire de ce conte une histoire de relations père/fils, mais cela ne modifie pas ce qui peut sembler être une relation simple entre récit, personnages, et sens. Mais la dimension communautaire, l'ensemble d'activités et de paroles par lesquels un auteur construit le contexte dans lequel une série d'événements trouve sa logique, est représentée par métonymie à travers la notion de sépulture, acte symbolique associant paroles, gestes et objet mémoriel (la pierre tombale qui marque à la fois le lieu, l'événement et, par l'inscription qu'elle porte, le déroulement de la vie ${ }^{18}$. L'absence d'une sépulture dans le conte constitue la trace d'autres absences, en particulier l'absence de mémoire, car Reuben n'est pas le seul à manquer au devoir de mémoire. La bataille de Lovewell, à l'époque d'Hawthorne, avait pris un statut légendaire, devenant le symbole du courage des premiers colons face à la résistance des Peaux-Rouges. Son camarade de classe, 
Longfellow avait consacré un poème à la gloire des combattants. Mais Hawthorne visiblement partageait l'opinion de ceux qui la voyaient comme un massacre inutile, prémisse de la sauvagerie avec laquelle les indigènes seraient tués pour laisser la place à la civilisation américaine ${ }^{19}$. L'espace qui sépare l'évocation de la bataille historique du début du récit de Roger Malvin marque d'autres blancs, d'autres ellipses dans le récit des débuts de la nation. Le narrateur, à la différence de celui qui raconte l'histoire d'Alice Doane, n'assure aucun lien spécifique entre le passé historique et le présent fictionnel, laissant la sépulture de Roger enterrer à la fois l'homme et le contexte dans lequel il avait trouvé sa véritée ${ }^{2}$. .Le lien entre contexte historique et univers fictionnel devra se construire à partir de la conscience des personnages, relayée par un narrateur qui fournit peu d'éléments pour établir la relation entre les gestes des personnages et leur environnement. Mais leurs pensées et leurs paroles permettent au lecteur d'entendre une partie du discours par lequel la petite communauté, synecdoque des premiers colons, construit son identité au fond de la forêt sauvage. Ce discours révèle un présent incertain déjà en partie transformé en légende. Roger incite l'autre à partir en lui expliquant que l'enterrement dans la forêt sauvage ne nécessite pas les mêmes rites que ceux pratiqués "dans les villes, et partout où vivent les hommes" (Hawthorne, 2007, 156). Il propose de graver sa propre pierre tombale et comme parcours de vie évoque son double statut de chasseur et de guerrier ${ }^{21}$. Non seulement Malvin officie-t-il à son propre enterrement, mais il accomplit une autre fonction pastorale en assurant Reuben que pour sa fille il sera "plus précieux qu'un père", évoquant « le long chemin plaisant que vous allez parcourir ensemble ", célébrant leurs noces à travers ses paroles (Hawthorne, 2007, 158). Comment pourrait-il parler ainsi s'il ne se fondait pas sur une confiance dans l'avenir de la communauté ancrée dans une culture partagée, construite à partir des histoires que les gens se racontaient sur leur destin providentiel dans le Nouveau Monde? Le pasteur John Higginson avait encouragé les protestants à fuir l'Europe en évoquant les montagnes vers lesquelles avait fui le peuple d'Israël (Pétillon dans Hawthorne, 2007, 622). À la différence de Longfellow, Hawthorne n'œuvrait pas à la construction d'une légende, mais cherchait à mesurer l'impact d'une légende déjà en partie formée. Lorsque Reuben demande si son compagnon ne s'est jamais trouvé dans la même situation, ce dernier lui raconte comment il a sauvé un camarade, et évoque un autre récit de la vie heureuse, reflet du rêve agricole développé plus tard par Jefferson, en expliquant que le camarade sauvé "se porte comme un charme et vit dans sa ferme, bien à l'abri des frontières " (Hawthorne, 2007, 160). À ce discours sur le bonheur pastoral s'en ajoute un autre, celui de la domesticité, par lequel le narrateur décrit Reuben en train de préparer son abandon de l'homme blessé en lui ramassant un petit stock de nourriture et en "prépar[ant] pour lui une nouvelle couche de feuilles de chêne sèches " (Hawthorne, 2007, 160). Comble de l'ironie, le vieil homme est décrit prodiguant des conseils au jeune, « comme s'il envoyait Reuben à la guerre ou à la chasse, tandis qu'il resterait en sécurité chez lui » (Hawthorne, 2007, 161). Le narrateur, tout en gardant sa distance, fait entendre les récits qui reflètent l'imaginaire collectif de la communauté et qui auraient dû rappeler à Reuben son devoir envers les autres. Le rocher sous lequel meurt Malvin, en rappelant le "roc de Plymouth» et le chêne, qui peut être associé au "Chêne de la Constitution", doublent ces récits officieux d'autres légendes, liées directement à la fondation de la Nation (Pétillon dans Hawthorne, 2007, 620). Mais la tradition de solidarité et de sacrifice représentée par Roger Malvin, que Reuben aurait dû entendre, lui échappe : «le désir de vivre, et l'espoir d'être heureux avaient repris 
leur empire sur son cœur, et il n'avait pas la force de leur résister » (Hawthorne, 2007, 162). Cette rupture entre le désir de bonheur et le devoir envers la communauté permet au lecteur de comprendre pourquoi Reuben symbolise aussi la surdité et l'oubli qui caractérisent l'Amérique d'Hawthorne, trop désireuse de glorifier le passé pour accepter sa complexité.

Dans ce conte, comme dans bien d'autres des meilleurs contes, Hawthorne travaille par focalisation plutôt que par le dialogue, laissant voir les paysages intérieurs qui sont le préciput, le dépôt visuel et sonore laissé par une tradition en partie orale. C'est la clé de leur résonance, bien au-delà des situations narratives. Reuben ne racontera jamais ce qui s'est réellement passé et interrogé par Dorcas, il la laissera finir la phrase qu'il n'arrive pas à prononcer et déclarer la mort du père. Reuben subit le sort de beaucoup des personnages d'Hawthorne, car coupé de toute participation réelle dans la communauté par un acte qu'il voit comme " un crime secret » (Hawthorne, 2007, 167), il passera sa vie à divaguer dans le paysage cauchemardesque de sa culpabilité. Tous les récits racontés par l'autre reviennent le hanter. Il va hériter de la ferme de Malvin, mais s'avérera un « laboureur négligent » (Hawthorne, 2007,168) et ne participera pas à la période de prospérité qui suivra la cessation des hostilités avec les Indiens. Pis, il deviendra, par le fait d'être dépourvu de l'énergie positive qui permet à ses compatriotes de réussir autour de lui, l'objet d'un certain nombre de procès, car le dernier rêve de l'Américain fondateur sera la création d'une société ordonnée, fondée sur le droit. Ne lui reste que la possibilité de faire un retour vers l'Éden qu'il a perdu en abandonnant celui qui avait défini par sa parole le paradis du Nouveau Monde. À la fin $\mathrm{du}$ conte, le narrateur se remet à construire dans la conscience de Dorcas et de son fils un paysage mental qui reflète la mythologie du pays. Dorcas s'affaire à domestiquer la forêt pendant que son fils accompagne son père à la chasse. Elle n'a pour seul divertissement que la lecture de « l'almanach du Massachusetts de l'année en cours » et d'une « vieille bible en caractères gothiques » (Hawthorne, 2007, 173). Alors que Reuben a déjà tiré la balle qui tuera son fils, le narrateur contemple longuement les travaux domestiques de Dorcas, associant la chanson qu'elle fredonne et en particulier les quatre vers du refrain avec «l'éclat du foyer dont ils disaient les joies» (Hawthorne, Idem, 176). La chanson exprime le lien entre la culture populaire, orale, et le travail de maitrise du pays sauvage accompli à travers la chasse, la guerre contre les Indiens, le travail de la terre, la domesticité et surtout "les joies du bonheur domestique» (Hawthorne, Ibid., 176).

17 Ce conte, l'un des premiers publiés par l'auteur, illustre bien la façon dont Hawthorne puisa dans les souvenirs d'une littérature orale et dans la réserve de légendes que l'Amérique avait construites pour justifier son destin. Son but n'était pas de fabriquer ses propres récits sur la base de cette littérature ni de renforcer la dimension réaliste de ses narrateurs en leur prêtant un parler oral. Hawthorne voyait la littérature orale comme un élément qui participait à la construction de l'imaginaire collectif au cœur duquel se dessinaient les parcours souvent solitaires et tragiques de ses personnages; elle donnait à cet imaginaire une résonance émotive qui était non seulement l'écho des récits précis qu'Hawthorne avait entendus, mais la trace de la charge émotionnelle liée aux peurs et aux désirs de toute une société en construction. Comme le souligne Alison Easton, les premiers contes, situés parfois dans des contextes politiques et idéologiques précis, "affichaient leur sujet comme relevant en partie du discours historique dans le sens où ils traçaient les processus par lesquels une société et un peuple se construisent, en montrant comment cette société fabrique l'histoire de ses 
origines» (Easton, 1996, 32, ma traduction). Les travaux des critiques comme Easton, qui abordent Hawthorne à travers son contexte culturel, permettent de distinguer entre l'imaginaire de l'auteur lui-même, nourri par sa conviction d'être un artiste solitaire, et la réserve de légendes et de récits à partir desquels il inventa des fables sur les rapports difficiles entre des personnages en quête de vérité personnelle et une société désireuse de façonner une mythologie collective. Au fond de la forêt dans laquelle Reuben Bourne abandonne Roger Malvin se niche un mensonge collectif dont l'erreur de Bourne est en partie le reflet, même si la société n'est pas responsable de son choix ${ }^{22}$. La société américaine du début du XIX siècle, à la recherche d'une stabilité sociale et intellectuelle, était en train de construire une identité collective à laquelle Hawthorne n'adhérait pas totalement. Il réussit à utiliser la forme du conte, en particulier son économie symbolique, pour traduire en termes narratifs la complexité d'une société déjà travaillée de l'intérieur par des contradictions héritées de l'époque des puritains et de celle de la Révolution.

Il serait possible de poursuivre cette analyse à travers d'autres contes et d'en trouver le prolongement dans les romances qu'il écrit à partir de la fin des années 1840. Il ne s'agit pas, comme nous l'avons constaté, de placer l'oralité au centre des préoccupations d'Hawthorne, mais de la traiter comme un composant de son univers imaginaire. Dans cette perspective, c'est la dimension affective du rapport de l'auteur à l'oralité du passé, la façon dont elle lui permettait d'explorer les peurs et les fantasmes de ses personnages, qui semble importante. C'est cet aspect de la tradition orale qu'Hawthorne traduisit dans ses contes, rendant ainsi encore plus obscur le fond de rêve, de peur et de désir contre lequel ses personnages accomplissent leur destin. En perturbant le lien entre l'individu et son entourage, ce que fait l'auteur dans tous ses récits, il ouvre un espace d'interprétation au sein de l'univers fictionnel, un gouffre de subjectivité dans lequel se précipitent les personnages. Prendre en compte l'impact d'une oralité vécue et entendue par l'auteur, c'est observer et mesurer la place de cette oralité parmi les éléments qui influencent la création de cet espace d'interprétation. Mais il s'agit également d'observer l'attitude d'Hawthorne vis-à-vis de la culture de son pays et de son passé, telle qu'elle s'exprime par la représentation de l'oralité. Cette approche permet de mettre en évidence la tradition qui lie Hawthorne à ses ancêtres puritains, pour qui la voix et la parole, comme relais de l'écriture biblique, furent les fondements de la communauté des chrétiens, mais pour qui la rumeur et la légende, autres manifestations de la parole, étaient des pièges qu'ils ne surent pas toujours éviter.

\section{BIBLIOGRAPHIE}

BAKER, Dorothy Z., 2007, America's Gothic Fiction: The Legacy of the Magnalia Christi Americana, Columbus, Ohio State University Press.

BELL, Michael David, 1971, Hawthorne and the Historical Romance of New England, Princeton, Princeton University Press. 
BERLANT, Lauren, 1991, The Anatomy of National Fantasy: Hawthorne, Utopia and Everyday Life, Chicago, University of Chicago Press.

CHANDLER, Elizabeth Lathrop, 1926, A Study of the Sources of the Tales and Romances Written by Nathaniel Hawthorne Before 1853, Smith College Studies in Modern Languages, vol. VII, $\mathrm{n}^{\circ} 4$.

CHARVAT, William, PEARCE, Roy Harvey, and SIMPSON, Claude M., 1974, The Centenary Edition of the Works of Nathaniel Hawthorne, vol. IX, Columbus, Ohio State University Press.

COHEN, Hennig, 1968, American Literature and American Folklore, in Coffin Tristram Potter, Our Living Traditions: An Introduction to American Folklore, New York, Basic Books, pp. 238-247.

DORSON, Richard, 1977, American Folklore, Chicago, University of Chicago Press.

EASTON, Alison, 1996, The Making of the Hawthorne Subject, Columbia and London, University of Missouri Press.

HAWTHORNE, Nathaniel, 1989, la Fille de Rappaccini et autres contes fantastiques, Paris, Librairie générale française [trad. par Henri Parisot, Françoise Charras et Charles Cestre].

HAWTHORNE, Nathaniel, 1992, Monsieur du miroir (et autres contes), Paris, José Corti [trad. par Pierre Leyris].

HAWTHORNE, Nathaniel, 1995a, Carnets Américains 1835-1853, Paris, José Corti [trad. et préfacé par Françoise Charras].

HAWTHORNE, Nathaniel, 1995b, le Faune de marbre, Paris, José Corti [trad. par Roger Kann].

HAWTHORNE, Nathaniel, 1995c, la Lettre écarlate, Paris, Presses Pocket [trad. par Charles Cestre].

HAWTHORNe, Nathaniel, 2007, Contes et récits, Arles, Actes Sud [trad. de l'américain par Muriel

Zagha, présentation et postface de Pierre-Yves Pétillon].

KESSELRING, Marion L., 1949, Hawthorne's Reading 1828-1850: A Transcription and Identification of Titles Recorded in the Charge-Books of the Salem Athenaeum, New York, The New York Public Library.

LABRIOLA, Patrick, 2004, Ludwig Tieck and Nathaniel Hawthorne: The Fairy Tale and the Popular Legend, The Journal of Popular Culture, vol. 38, $\mathrm{n}^{\circ}$ 2, pp. 325-332.

LUEDTKE, Luther S., 1989, Nathaniel Hawthorne and the Romance of the Orient, Bloomington, Indiana University Press.

LUNDBLAD, Jane, 1965, Nathaniel Hawthorne and European Literary Tradition, New York, Russell and Russell.

MARLER, Robert F., 1974, From Tale to Short Story: The Emergence of a New Genre in the 1850's, American Literature, vol. 46, $\mathrm{n}^{\circ}$ 2, pp. 153-169.

MATHER, Cotton, 1853, Magnalia Christi Americana or the Ecclesiastical History of New England from its first planting, in the year 1620, unto the year of our lord 1698, t. II, Hartford, Silas Andrus and Son. MELLOW, James Robert, 1980, Nathaniel Hawthorne in his Times, Boston, Houghton Mifflin. MILDER, Robert, and FULLER, Randall(eds), 2009, The Business of Reflection: Hawthorne in His Notebooks, Columbus, Ohio State University Press.

PORTELLI, Alessandro, 1994, The Text and the Voice: Writing, Speaking and Democracy in American Literature, New York, Columbia University Press.

REID, Margaret, 2004, Cultural Secrets as Narrative Form: Storytelling in Nineteenth-century America, Columbus, Ohio State University Press. 
STEWART, Randall, 1932, The American Notebooks by Nathaniel Hawthorne, Based upon the Original Manuscripts in the Pierpont Morgan Library, New Haven, Yale University Press.

WINEAPPLE, Brenda, 2003, Hawthorne: A Life, New York, A. Knopf.

WOODSON, Thomas, L. SMITH, Neal and PEARSON, Norman Holmes (eds), 1984, Hawthorne's

Correspondence: The Letters 1813-1843, Columbus, Ohio State University Press.

\section{NOTES}

1. Portelli explique que dans la Lettre écarlate « l'éruption de la voix disperse systématiquement la vérité 'littérale' et l'autorité documentaire, la certitude historique et la crédibilité narrative » (1994, 32, ma traduction).

2. Cette approche est représentée par des ouvrages tels que ceux de David REYNOLDS, Beneath the American Renaissance: The Subversive Imagination in the Age of Emerson and Melville (1989), de Lauren BERLANT, The Anatomy of National Fantasy: Hawthorne, Utopia and Everyday Life (1991), d'Alison EASTON, The Making of the Hawthorne Subject (1996) et de Margaret REID, Cultural Secrets as Narrative Form: Storytelling in Nineteenth-century America (2004).

3. La chronologie de la composition et de la publication des contes et esquisses d'Hawthorne a été établie minutieusement par les éditeurs et critiques de son œuvre. Elle constitue en ellemême un récit de la vie de l'auteur et de ses rapports complexes avec lui-même, avec ses proches, ses éditeurs et le public qu'il cherchait, récit dans lequel on peut lire également sa relation compliquée avec son époque. Le travail d'Elizabeth Lathrop CHANDLER dans A Study of the Sources of the Tales and Romances Written by Nathaniel Hawthorne Before 1853 (1926) a été particulièrement important de ce point de vue. La publication du Centenary Edition des œuvres d'Hawthorne permit de faire le point de cette question et de rassembler les informations dans les explications qui accompagnent les textes.

4. Dans une étude de l'émergence de la nouvelle comme genre dans la littérature américaine du $\mathrm{XIX}^{\mathrm{e}}$ siècle, Robert F. Marler fait remarquer que Melville, qui écrivait à la fois des contes (tales) et des nouvelles (short stories) utilisa un narrateur oral emprunté à la tradition du tall tale (un genre oral faisant largement appel à l'exagération, d'où le mot «tall ») à des fins réalistes dans un chapitre de Moby Dick intitulé "The Town-Ho's Story" (1974). D'après Marler, Hawthorne, comme Melville, abandonna peu à peu le conte, avec ses personnages stéréotypés, son sentimentalisme et son didactisme pour une approche plus nuancée de personnages puisés dans la vie réelle sous la pression des éditeurs des revues dans lesquelles ils publiaient leur fiction, éditeurs qui, eux, étaient soucieux des goûts de leur lectorat.

5. C'est Nathaniel Hawthorne qui ajouta un «w" à son nom de famille peu après avoir quitté l'université (Mellow, 1980, 10).

6. Les vieux personnages rencontrés dans le bureau de la douane auraient fourni à Hawthorne la matière de son célèbre prologue à la Lettre écarlate, dans lequel il crée le contexte de la découverte de la lettre brodée et invente l'histoire qui s'ensuit. Il évoque le don pour la narration de l'un des inspecteurs, déclarant que s'il avait pu préserver «la vigueur pittoresque de son style » en reprenant ses histoires, il aurait pu créer "quelque chose de neuf en littérature » (Hawthorne, 2007, 535). Cette attitude est bien sûr une posture narrative, mais elle révèle néanmoins sa conscience de la narration orale comme l'une des voies pour «créer l'apparence d'un monde avec de la matière aérienne" (Hawthorne, Idem, 535). La relation entre la vie quotidienne, matérielle, réelle et le monde impalpable de l'imagination constituait l'une des préoccupations majeures de l'écrivain. Comme nous le verrons au cours de cette démonstration, 
la dimension orale de la narration participait à la fois à la création d'une distance légendaire et à l'évocation d'une proximité avec une expérience vécue.

7. Essentiel de ce point de vue est l'ouvrage de Marion L. Kesselring (1949). Kesslering a noté tous les titres qui ont été empruntés par Hawthorne avec la carte de bibliothèque de sa tante Mary. La liste est impressionnante et contient non seulement les classiques de la littérature anglaise, mais également de nombreux auteurs français comme Montaigne, Corneille, Racine, Voltaire et Rousseau.

8. Il y a de nombreuses allusions à ce livre dans ses contes et il a fait partie de sa bibliothèque personnelle jusqu'à la fin de sa vie (Luedtke, 1989, 52).

9. Mellow parle de sa lecture des Voyages de Hakluyt, des Notes on the West Indies de Pinckard, de Travels in Various Parts of Peru de Temple et de Travels in India d'Herbert (Mellow, 1980, 41). Luedtke pour sa part fournit une liste complète des livres ayant pour sujet l'Orient empruntés par Hawthorne au Salem Atheneum (Luedtke, 1989, 223-26).

10. L'un des passages les plus célèbres dans lesquels Hawthorne aborde sa relation avec la réalité américaine se trouve dans la préface de son roman le Faune de marbre. Dans ce passage Hawthorne compare l'Italie, terrain propice à l'imagination, à son propre pays, caractérisé par « une vulgaire prospérité qui se montre en plein jour» et qui ne lui inspire aucune intrigue comportant une part de mystère ou d'ombre (Hawthorne, 1995b, 9).

11. D'après Chandler, Hawthorne aurait lu les contes à sa mère en 1825. Chandler fait l'hypothèse, sur la base des sujets et du style, que les contes «An Old Woman's Tale » et «The Hollow of the Three Hills », publiés dans la Salem Gazette en 1828 et 1830, faisaient partie, comme celui qui s'appelait "Alice Doane » et qui a été repris sous le titre d' "Alice Doane's Appeal », de ce premier recueil (Chandler, 1926, 7-12).

12. "The Gentle Boy», "The Wives of the Dead", "My Kinsman, Major Molineux», et "Roger Malvin's Burial » furent publiés de façon anonyme dans The Token de 1832 (Luedtke, 1989, 104).

13. Les critiques ne sont pas d'accord concernant la composition exacte du recueil, tel qu'il était prévu par Hawthorne. Les éditeurs de la Centenary Edition considèrent que les dix-sept morceaux publiés dans le New England Magazine entre novembre 1834 et décembre 1835 faisaient partie du projet original. Ils évoquent la possibilité que d'autres textes, publiés dans The American Monthly et The Token en faisaient également partie (Charvat, Pearce, Simpson, 1974, 495). Luedtke y inclut, en plus des textes mentionnés dans la Centenary Edition, «The Seven Vagabonds » et "Fragments from the Journal of a Solitary Man », qui logiquement constituent le cadre narratif qui relie les autres textes, bien qu'ils aient paru dans d'autres revues (Luedtke, 1989, 106).

14. La source du célèbre "Rip Van Winkle" fut l'histoire de Peter Klaus trouvée dans le Volkssagen d'Otmar (Cohen, dans Coffin, 1968, 238-247, 245). À noter aussi le fait qu'Edgar Allen Poe accusa Hawthorne d'avoir imité les contes de l'Allemand Ludwig Tieck. Patrick Labriola fait remarquer qu'en dépit du manque de preuves d'une influence directe de Tieck, on peut constater que les deux auteurs utilisent les contes de fées et les légendes populaires pour créer un effet de surnaturel (Labriola, 2004, 325-332).

15. Le conte, comme plusieurs critiques l'ont fait remarquer, est fortement empreint d'éléments gothiques. Lundblad identifie au moins cinq éléments gothiques (Lundblad, 1965, 91). Lauren Berlant considère que la dimension gothique traduit l'excès d'affect lié aux «formes sociales du pouvoir", ce pouvoir étant au centre des préoccupations de l'auteur dans sa représentation du passé (Berlant, 1991, 35, ma traduction).

16. Parmi les voix qu'Hawthorne entendait dans sa tête, il y avait celle de Cotton Mather. Mather était un homme de l'Église, et donc un homme de l'écrit, profondément habité par le texte biblique. Mais la Magnalia Christi Americana n'est pas seulement l'histoire de la NouvelleAngleterre, de ses gouverneurs et magistrats, de ses lois, etc. L'histoire racontée par Mather contient également un grand nombre d'histoires qui relèvent du folklore et de la légende, dont les sources ne sont pas réellement documentées. Mather puisa dans les histoires racontées et 
transmises par le peuple, se servant de ce fonds oral pour élaborer la légende du statut exceptionnel du peuple de la colonie de Massachusetts. Richard Dorson évoque les contes folkloriques qui ont été inscrits dans le compte-rendu du tribunal pendant le procès de Susanna Martin en juin 1692 (Dorson, 1977, 37).

17. "The Great Carbuncle » est un exemple de cette approche. Michael Bell parle de la façon dont Hawthorne associe les « possibilités imaginatives » d'une légende indienne avec le paysage du Maine (Bell, 1971, 197).

18. Le rapport est moins évident en anglais, car le titre en anglais comporte le mot « burial », qui signifie l'acte d'enterrer quelqu'un mais pas la pierre tombale.

19. Dans sa biographie de l'auteur, Brenda Wineapple donne quelques détails sur les circonstances autour de l'écriture de ce conte, dont Hawthorne eut probablement l'idée en 1825, l'année où il termina ses études à Bowdoin. Ce fut aussi l'année où le pays célébra le centenaire de cette fameuse bataille, présentée comme un acte de courage. Mais en réalité il s'agissait du massacre d'une dizaine d'Indiens Pequawket par le capitaine John Lovewell et quarante-six de ses hommes. À la suite de cette bataille, les troupes de Lovewell furent prises en embuscade par un grand nombre d'Indiens et dans leur retraite précipitée elles abandonnèrent trois soldats qu'elles croyaient morts (Wineapple, 2003, 67-68). Pierre-Yves Pétillon fait la même analyse dans son introduction à la traduction des Contes et récits, considérant que ce conte, comme d'autres écrits à cette époque, peut être lu comme « une 'déconstruction' ironique de cette version officielle de l'histoire » (Pétillon dans Hawthorne, 2007, 30).

20. Le narrateur, dans le paragraphe du début, évoque «l'héroïsme de la petite troupe qui affronta une armée deux fois plus nombreuse en plein cœur même du pays ennemi » (Hawthorne, 2007, 153). Mais il enveloppe l'histoire racontée dans la transmission orale d'incidents : «Ceux qui entendirent conter, de la bouche de vieillards, la destinée des quelques soldats en état de battre en retraite, après 'la bataille de Lovell' sauront reconnaître, à travers des noms fictifs, certains des épisodes évoqués dans les pages suivantes (Hawthorne, Idem, 153-154).

21. Il $\mathrm{y}$ aurait beaucoup à dire sur cette double vocation, déjà un constituant essentiel de la légende américaine. Elle contribue fortement au dénouement tragique, car c'est en chassant que Reuben deviendra, à son insu, guerrier et meurtrier de son propre fils. Pétillon évoque à propos de l'importance de la terre sauvage (wilderness) « la triade indo-européenne de Dumézil : orator, laborator, bellator. Le wilderness est le lieu de l'orator, qui s'y retire pour prier ; le lieu du laborator, qui le défriche avec sa charrue ; et enfin le lieu du bellator, le guerrier-chasseur qui y poursuit son ennemi, Indien, ours ou bison» (Pétillon dans Hawthorne, 2007, 623).

22. Easton considère que c'est le besoin de la communauté de croire en l'héroïsme des soldats qui ont participé à la bataille de Lovewell qui crée la pression qui empêche Bourne d'avouer son mensonge (Easton, 1996, 50).

\section{RÉSUMÉS}

Nathaniel Hawthorne ne vient pas immédiatement à l'esprit lorsqu'on évoque le rôle de l'oralité dans le développement de la littérature américaine au $\mathrm{XIX}^{\mathrm{e}}$ siècle. Pourtant les légendes et histoires qu'il entendit dans son enfance, en particulier celles ayant un rapport avec le passé de sa propre famille à l'époque des puritains, jouent un rôle important dans son imaginaire. En observant de près la façon dont l'auteur représente le passé légendaire de l'Amérique, en 
particulier dans les premiers contes, on peut comprendre comment cette conscience d'une littérature orale a influencé sa représentation de l'Amérique à une époque où le pays était en train d'élaborer une mythologie nationale.

Nathaniel Hawthorne is not often perceived as a writer concerned with the use of orality as a means of developing the specificity of American literature. However oral traditions, particularly those that related him to the past of his Puritan ancestors, constitute an important part of his literary background. By observing Hawthorne's description of American life in his notebooks and the way in which he represented the Puritan and Revolutionary past in his early tales, one can measure his interest in oral legends and his desire to examine the conditions in which those legends contributed to the construction of a national identity in the early nineteenth century.

\section{INDEX}

Thèmes : littérature orale

Mots-clés : Hawthorne Nathaniel (1804-1864), littérature américaine du xixe siècle, folklore américain

Keywords : Hawthorne Nathaniel (1804-1864), Nineteenth-century Americain Literature, American Folklore

\section{AUTEUR}

\section{KATHIE BIRAT}

Université de Lorraine, IDEA 